\title{
On Neural Networks
}

\section{I am only a layman in the neural network space so the ideas and opinions in this column are sure to be refined by comments from more knowledgeable readers.}

The recent successes of multilayer neural networks have made headlines. Much earlier work on what I imagine to be single-layer networks proved to have limitations. Indeed, the famous book, Perceptrons, ${ }^{\mathrm{a}}$ by Turing laureate Marvin Minsky and his colleague Seymour Papert put the kibosh (that's a technical term) on further research in this space for some time. Among the most visible signs of advancement in this arena is the success of the DeepMind AlphaGo multilayer neural network that beat the international grand Go champion, Lee Sedol, four games out of five in March 2016 in Seoul. ${ }^{\text {b }}$ This was followed by a further success against Ke Jie winning three games of three in Wuzhen in May 2017. ${ }^{\mathrm{c}}$ Further developments have led to AlphaGo Zero ${ }^{d}$ that learned to play championship Go in only 40 days with only the rules of Go and a reinforcement learning algorithm to generate the neural network weightings. AlphaGo Zero learned to play chess well enough to beat most (maybe all?) computerbased players in 24 hours. ${ }^{\mathrm{e}}$

These developments are dependent in part on vastly faster and cheaper computing engines such as graphical processing units and, at Google, tensor processing units that run multilayer ma-

a https://mitpress.mit.edu/books/perceptrons

b https://en.wikipedia.org/wiki/AlphaGo_ versus_Lee_Sedol

c https://en.wikipedia.org/wiki/AlphaGo_ versus_Ke_Jie

d https://deepmind.com/blog/alphago-zerolearning-scratch/

e https://en.chessbase.com/post/the-future-ishere-alphazero-learns-chess chine learning algorithms swiftly and increasingly efficiently. Google used a version of its tensor processing system, TensorFlow, to reduce the cost of cooling a datacenter by $40 \% .{ }^{\mathrm{f}}$ Many other applications are surfacing for these neural network systems, including reliable identification of diseases (for example, diabetic retinopathy, cancerous cells), situational awareness for self-driving cars, and a raft of other hard recognition and optimization problems.

Speculation is rampant as to where these methods may take us in the future. Powerful decision-making tools are in development or already in operation, absorbing and applying large amounts of data to discrimination tasks normally reserved for human judgment. Therein lies an issue deserving of the attention of ACM members and all others engaged in fashioning these new tools. These systems are brittle in some respects. The training sets used to develop the neural network weights can be biased in ways not known to the trainers. The choices or decisions indicated by the networks can also exhibit chaotic effects in the sense that small changes in inputs can result in extreme changes in output. In socalled generative adversarial networks, ${ }^{\mathrm{g}}$ two networks are pitted against each other (see Goodfellow et al. p. 56). One tries to fool the other into thinking that an image of a dog, for example, is actually a cat. Changes to small numbers of

f https://deepmind.com/blog/deepmind-aireduces-google-data-centre-cooling-bill-40/

$\mathrm{g}$ https://deeplearning4j.org/generativeadversarial-network pixels that are imperceptible to humans can cause a neural network to make major classification mistakes. In my layman's cartoon model of this phenomenon, I imagine each pixel in the input image is a distinct dimension in a high dimension space. Hyperplanes separate images of animals, for instance, from each other. Small changes in the values of some pixels may cause a vector in hyperspace to move across the hyperplane boundary and cause the system to misidentify an image of a dog as a cat or something else.

These hazards drive the need for serious thinking about the potential to depend too much on the output of such neural systems or to make autonomous decisions without human intervention. Attention to the training sets and assiduous testing against a wide range of inputs seems essential to limit serious side effects of decision making using these systems. That such neural networks can be extremely valuable is inarguable in the face of demonstrated results so far. That they can also be catastrophically wrong is equally evident, triggering a serious need for ethical considerations in their development and application. This is true in general for all software, but especially so for neural networks which functions are still somewhat mysterious and which successes are so spectacular that it is tempting to ignore the potential for unintended consequences of their use. $\quad$ c

Vinton G. Cerf is vice president and Chief Internet Evangelist at Google. He served as ACM president from 2012-2014.

Copyright held by author 